
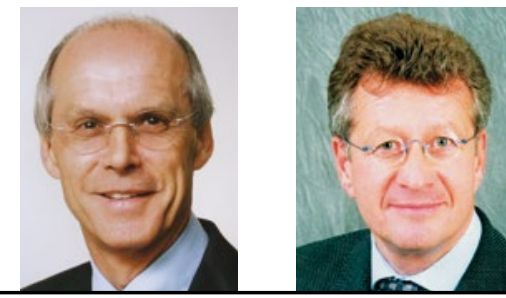

Dr. med. P. Stiefelhagen

Internist,

Hachenburg

\title{
Oligomenorrhö
}

\section{Kann es ein polyzystisches Ovarialsnydrom sein?}

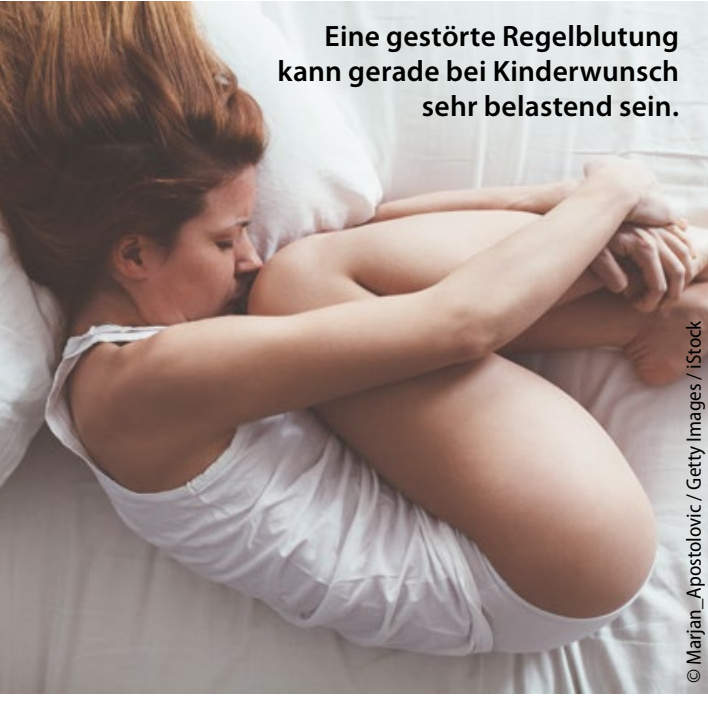

$?$ Dr. Claudia Petroni, Brixen/Südtirol: Meine 24-jährige Patientin leidet seit Beginn der Menarche an Oligomenorrhö bzw. Amenorrhöphasen. Hirsutismus oder Gewichtszunahme liegen nicht vor. Laborproben aus dem Jahr 2013 mit Hormonuntersuchungen und Nüchternglukose zeigten sich durchwegs normal, speziell auch die Spiegel von $\mathrm{LH}, \mathrm{FSH}$, Testosteron und TSH. Lediglich die Konzentration von Thyreoglobulinantikörpern war erhöht. Könnte dies eine Relevanz haben?

Die Patientin erhielt aufgrund des hohen Leidensdrucks über Jahre ein orales biphasisches Kontrazeptivum, mit dem es ihr sehr gut ging. Nun besteht aber Kinderwunsch, und wir wollen der Sache auf den Grund gehen. Nach Absprache hat sie die Pille abgesetzt. Am Zyklustag 31 hat noch keine Regelblutung eingesetzt. Die Patientin macht auch Temperaturmessungen, die eine gezackte, unregelmäßige Kurve ergeben.

Eine Gynäkologin hat einmal den Verdacht auf ein polyzystisches Ovarialsyndrom (PCOS) geäußert, ohne dass mir ein Beweis dafür vorläge. Wäre das eine Möglichkeit? Ich würde die Patientin nochmals zum Gynäkologen schicken. Sollte ich ihr in der Zwischenzeit ein Progesteronpräparat geben, um die Gelbkörperphase zu stabilisieren?

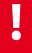

MMW-Experte Stiefelhagen: Ich empfehle Ihnen, die Patientin nochmals in einer speziellen gynäkologischendokrinologischen Sprechstunde mit der gezielte Fragestellung nach einem PCOS vorzustellen. Vorher würde ich keine Hormonbehandlung einleiten. Wenn sich ansonsten keine Hinweise für eine Schilddrüsenfunktionsstörung ergeben, wofür der TSH-Normwert spricht, und auch keine Hinweise für eine Thyreoiditis vorliegen, würde ich der isolierten Erhöhung der Tyreoglobulinantikörper keine Bedeutung beimessen.
I MMW-Experte Füeßl: Die klinischen Zeichen des PCOS wie Hirsutismus, Akne oder androgenetische Alopezie liegen bei der Patientin offensichtlich nicht vor. Das macht die Diagnose schon einmal unwahrscheinlich, wenngleich bei Zyklusunregelmäßigkeiten eine Fülle von Ursachen in Betracht kommt. Wie viele Zysten in den Ovarien tatsächlich vorhanden sind, scheint im vorliegenden Fall noch nicht zuverlässig bekannt zu sein. Insofern lohnt sich immer eine gynäkologische Zweitmeinung mit suffizienter sonografischer Diagnostik. Laut Definition liegt ein PCOS bei mindestens zwölf Follikeln mit jeweils einem Durchmesser von 2-9 mm oder einem Ovarvolumen von mindestens $10 \mathrm{ml}$ vor.

Eine neuerliche Hormondiagnostik sollte FSH, LH, Prolaktin, Testosteron, Androstendion, DHAES, die wiederholte Bestimmung von Progesteron an den Zyklustagen 22-24 zum Nachweis einer Anovulation, Insulin und TSH umfassen.

Die Thyreoglobulinantikörper sollte man vergessen. Die Gabe eines Progesteronpräparats in der zweiten Zyklushälfte würde ich erst nach den mehrfachen Progesteronbestimmungen vornehmen und im Übrigen auch dem Gynäkologen überlassen. Mit diesem Programm haben Sie als Hausärztin mehr als genug getan. 